\title{
Blood pressure at hospital admission and outcome after primary intracerebral hemorrhage
}

\author{
Erwin Chiquete'1, Ana Ochoa-Guzmán², Ángel Vargas-Sánchez², Jorge Navarro-Bonnet ${ }^{2}$, \\ Miquel A. Andrade-Ramos ${ }^{2,3}$, Patricia Gutiérrez-Plascencia², José L. Ruiz-Sandoval2,4
}

\author{
${ }^{1}$ Neurology and Psychiatry Department, Instituto Nacional de Ciencias Medicas \\ y Nutricion "Salvador Zubiran", Guadalajara, Mexico \\ 2Department of Neurology, Hospital Civil de Guadalajara "Fray Antonio Alcalde", \\ Guadalajara, Mexico \\ ${ }^{3}$ Department of Internal Medicine, Hospital Civil de Guadalajara "Fray Antonio \\ Alcalde", Guadalajara, Mexico \\ ${ }^{4}$ Department of Neurosciences, Centro Universitario de Ciencias de la Salud, \\ Universidad de Guadalajara, Guadalajara, Mexico
}

Submitted: 29 March 2012

Accepted: 25 September 2012

Arch Med Sci 2013; 9, 1: 34-39

DOI: $10.5114 /$ aoms.2013.33346

Copyright $\odot 2013$ Termedia \& Banach

\begin{abstract}
Introduction: The importance of the admission blood pressure (BP) for intracerebral hemorrhage $(\mathrm{ICH})$ outcome is not completely clear. Our objective was to analyze the clinical impact of BP at hospital arrival in patients with primary $\mathrm{ICH}$. Material and methods: We studied 316 patients (50\% women, mean age: 64 years, $75 \%$ with hypertension history) with acute primary ICH. The first BP reading at admission was evaluated for its association with neuroimaging findings and outcome. A Cox proportional hazards model and Kaplan-Meier analyses were constructed to evaluate factors associated with in-hospital mortality.

Results: Intraventricular irruption occurred in $52 \%$ of cases. A high frequency of third ventricle extension was observed in patients with BP readings in the upper quartiles of the distribution (systolic, diastolic, or mean arterial pressure). Blood pressure readings did not correlate with hematoma volumes. In-hospital case fatality rate was $46 \%$ (63\% among those with ventricular irruption). Systolic BP (SBP) > $190 \mathrm{~mm} \mathrm{Hg}$ was independently associated with in-hospital mortality in supratentorial $(n=285)$ ICH (hazard ratio: $1.19,95 \%$ confidence interval: 1.02-1.38, for the highest vs. the lowest quartile) even after adjustment for known strong predictors (age, ICH volume, Glasgow coma scale and ventricular extension). Blood pressure was not significantly associated with ventricular extension or outcome in patients with infratentorial ICH.

Conclusions: A high BP on admission is associated with an increased risk of intraventricular extension and early mortality in patients with supratentorial $\mathrm{ICH}$. However, a significant proportion of patients with high BP readings without ventricular irruption still have an increased risk of death.
\end{abstract}

Key words: blood pressure, diastolic, hematoma, intracerebral hemorrhage, hypertension, outcome, systolic.

\section{Introduction}

Hypertension is the single most important risk factor for primary intracerebral hemorrhage $(\mathrm{ICH})$ [1-6]. On admission to hospital most patients have the antecedent of hypertension, but nearly $10 \%$ correspond to new cases diagnosed after the brain hemorrhage has occurred [2-6]. It is well known that blood pressure (BP) readings are higher after

\section{Corresponding author:}

Dr. José Luis Ruiz Sandoval Servicio de Neurología Hospital Civil de Guadalajara "Fray Antonio Alcalde" Hospital 278, Guadalajara Jalisco, México C.P. 44280 Phone: 52 (33) 36134016 Fax: 52 (33) 36141121 E-mail: jorulej-1nj@ prodigy.net.mx 
acute cerebrovascular disease, as compared with the basal state [7], so a high BP in the acute state can be both the cause and the consequence of a non-traumatic brain hematoma $[2,8]$. Nevertheless, the clinical relevance of BP readings upon arrival at the Emergency Department for radiological characteristics and outcome after $\mathrm{ICH}$ is not completely clear [8-11].

The purpose of this study was to analyze the clinical significance of the first $B P$ reading performed at hospital admission in patients with acute primary $\mathrm{ICH}$, in relation to neuroimaging characteristics and short-term outcome. Our hypothesis was that a high BP is associated with short-term mortality, mainly through extension into the ventricular system. This may help to define adequate patient selection for acute tight BP control after ICH. Our findings are relevant in view of the recent clinical trials on acute control of BP in patients with primary cerebral hematomas.

\section{Material and methods}

\section{Patient characteristics}

This is a descriptive study on patients prospectively included in a single-center research database [10]. The internal Committee of Ethics of our hospital approved the present study. Informed consent was obtained from the patients or their closest relative. We reviewed 316 consecutive adult patients hospitalized in the general wards of our institution with acute primary $\mathrm{ICH}$. Inclusion criteria were: 1 ) $\mathrm{ICH}$ was defined as a sudden focal neurological deficit explained by a brain hematoma demonstrated in head computed tomography (CT) or magnetic resonance imaging (MRI) performed in the first $48 \mathrm{~h}$ after arrival; 2) supratentorial or infratentorial parenchymal hematomas; and 3) having available the first BP readings at hospital admission to the Emergency Department registered within the first $24 \mathrm{~h}$ after symptoms onset. The exclusion criteria were: 1) hematomas secondary to head trauma, arteriovenous malformations, aneurysms, vasculitis, cerebral vein and sinus thrombosis, neoplasms, hemorrhagic diathesis, anti-coagulant therapy or illicit drug abuse; 2) time from symptoms onset, neuroimaging or outcome information not available. Blood pressure readings were performed immediately following arrival at the Emergency Department, and before exposure to any anti-hypertensive therapy [12]. After a systolic BP (SBP) $>200$ $\mathrm{mm} \mathrm{Hg}$ or a mean arterial pressure $($ MAP) $>150$ $\mathrm{mm} \mathrm{Hg}$ was identified, aggressive BP control was applied, as recommended [7]. A standardized structured questionnaire was used to collect information on risk factors, neuroimaging characteristics, acute management, in-hospital complications and outcome at discharge.
A hypertensive etiology was defined as the patient having a history of hypertension or SBP $>140 \mathrm{~mm} \mathrm{Hg}$ or diastolic BP (DBP) > $90 \mathrm{~mm} \mathrm{Hg}$ maintained throughout one week or more after the hospital admission, in the absence of other potential causes [3, 4]. Intracerebral hemorrhage volume was calculated by analysis of the head CT scans according to the $A B C / 2$ method. The first SBP and $D B P$ reading taken on arrival at the Emergency Department, before anti-hypertensive intervention, was used to calculate MAP, as MAP $=\mathrm{DBP}+0.412$ (SBP - DBP) [3, 4]. This MAP formula corrects for the spurious variance of calculated MAP seen in individuals with hypertension; thus, it is the ideal method in cases with hypertensive $\mathrm{ICH}$.

\section{Statistical analysis}

Pearson $\chi^{2}$ and Fisher exact test were used to assess nominal variables in bivariate analyses. Pearson correlation was used in continuous variables. The SBP, DBP and MAP were evaluated for their association with relevant neuroimaging findings and short-term outcome. The distributions of SBP, DBP and MAP values were divided into quartiles to compare the highest (Q4: >190,110, 135 and $85 \mathrm{~mm} \mathrm{Hg}$; respectively) vs. lower (Q1-Q3) or the lowest (Q1) quartiles for their clinical correlates. To test the association of BP values with short-term mortality, multivariate Cox proportional hazards models were constructed for the entire cohort and for the cases with supratentorial ICH $(n=285)$ separately. Input variables were those significantly associated with mortality in bivariate analyses. Actuarial analyses by Kaplan-Meier survival curves were constructed to evaluate the prognostic association of SBP with mortality observed after hospital admission. Log-rank tests were used to compare Q4 and Q1 in actuarial curves. All $p$ values are two-sided and considered significant when $p<0.05$. Except for the multivariable analysis, which implies adjustments for relevant confounders, a Bonferroni correction (multiple-comparison adjustment) was applied to all significant associations in bivariate analyses, with a correction factor derived from the number of independent variables tested for each outcome of interest (ICH location, BP estimate and mortality). SPSS (SPSS Inc., Chicago, IL.) v17.0 statistical package was used for all calculations.

\section{Results}

We studied 316 patients (mean age: 64 years, range: 18 to 99 years) with primary $\mathrm{ICH}: 159$ (50.3\%) women (mean age: 65 years, range: 18 to 99 years) and 157 (49.7\%) men (mean age: 63 years, range: 18 to 95 years). No relevant differences were observed according to gender, except for more cases with the antecedent of hypertension in women, 
as compared with men (74\% vs. $60 \%$, respectively; $p=0.009$ ).

Systolic BP > $190 \mathrm{~mm} \mathrm{Hg}$ was more frequently found among older patients than those aged $<45$ years, but not significantly after Bonferroni correction (Table I). Irruption into the third ventricle was more common among patients with the highest (i.e., Q4) BP estimates (Table I) than those with a lower BP. Patients with SBP $>190 \mathrm{~mm} \mathrm{Hg}$ and $\mathrm{DBP}>110 \mathrm{~mm} \mathrm{Hg}(\mathrm{BP}>190 / 110 \mathrm{~mm} \mathrm{Hg})$ had more extension into the third ventricle, as compared with patients with SBP > $190 \mathrm{~mm}$ Hg but with DBP $\leq 110 \mathrm{~mm} \mathrm{Hg}$ (56\% vs. 30\%, respectively; Bonferroni $p=0.04)$. No significant correlation was found between BP (either as quartiles or crude values) and hematoma volume. In cases with supratentorial $\mathrm{ICH}(n=285)$, intraventricular extension occurred more frequently in those with deep rather than lobar hematomas ( $59 \%$ vs. $38 \%$, respectively; Bonferroni $p=0.01$ ).

In patients with supratentorial ICH, $46 \%$ with lobar and $26 \%$ with deep hematomas underwent surgical evacuation $(p=0.04)$. Conversely, ven- triculostomy was significantly more common among patients with deep ICH than in lobar ICH cases ( $11 \%$ vs. $1 \%, p=0.005)$. High BP estimates were not associated with the probability of receiving any surgical intervention. Median hospital stay was 9 days (range: 1 to 82 days). Ten (3.2\%) patients were discharged with total recovery, $45(14.2 \%)$ with moderate disability, 110 (34.8\%) with severe disability and 7 (2.2\%) in vegetative state. The in-hospital case fatality rate was $45.6 \%$ $(n=144)$, with $4.4 \%$ of in-hospital deaths occurring within the first $48 \mathrm{~h}$ after admission. In-hospital mortality was higher among patients with SBP $>190 \mathrm{~mm} \mathrm{Hg}$ (Table I), and in cases with any extension into the ventricular system, in bivariate analyses. Moreover, BP measures were not significantly associated with acute case fatality or with extensions into the ventricles in patients with infratentorial ICH. On the other hand, in a Cox proportional hazards model applied to patients with supratentorial ICH, the highest quartile of SBP was independently associated with a high frequency of acute case fatalities, after adjusting for known pre-

Table I. Clinical characteristics of 316 patients with ICH, according to blood pressure readings

\begin{tabular}{|c|c|c|c|c|c|c|}
\hline \multirow[t]{2}{*}{ Variables } & \multicolumn{2}{|c|}{ SBP } & \multicolumn{2}{|c|}{ DBP } & \multicolumn{2}{|c|}{ MAP } \\
\hline & $\begin{array}{c}190 \mathrm{~mm} \mathrm{Hg} \\
(\mathrm{Q} 4) \\
(n=77)\end{array}$ & $\begin{array}{c}\leq 190 \mathrm{~mm} \mathrm{Hg} \\
(\mathrm{Q} 1-\mathrm{Q} 3) \\
(n=239)\end{array}$ & $\begin{array}{c}>110 \mathrm{~mm} \mathrm{Hg} \\
(\mathrm{Q} 4) \\
(n=54)\end{array}$ & $\begin{array}{c}\leq 110 \mathrm{~mm} \mathrm{Hg} \\
(\mathrm{Q} 1-\mathrm{Q} 3) \\
(n=262)\end{array}$ & $\begin{array}{c}135 \mathrm{~mm} \mathrm{Hg} \\
(\mathrm{Q} 4) \\
(n=75)\end{array}$ & $\begin{array}{c}\leq 135 \mathrm{~mm} \mathrm{Hg} \\
(\mathrm{Q} 1-\mathrm{Q} 3) \\
(n=241)\end{array}$ \\
\hline GCS at arrival $\leq 8, n(\%)$ & $29(37.7)$ & $63(26.4)$ & $20(37.0)$ & $72(27.5)$ & $26(34.7)$ & $66(27.4)$ \\
\hline $\mathrm{ICH}$ volume > $70 \mathrm{ml}, n$ (\%) & $13(16.9)$ & $25(10.5)$ & $8(14.8)$ & 30 (11.5) & $12(16.0)$ & $26(10.8)$ \\
\hline Supratentorial, $n$ (\%) & $65(84.4)$ & $220(92.1)$ & $46(85.2)$ & $239(91.2)$ & $62(82.7)$ & $223(92.5)$ \\
\hline Lobar & $9(13.8)$ & $77(35.0)^{a}$ & $3(6.5)$ & $83(34.7)^{\mathrm{a}}$ & $5(8.1)$ & $81(36.3)^{a}$ \\
\hline Deep & $56(86.2)$ & $143(65.0)$ & $43(93.5)$ & $156(65.3)^{a}$ & $57(91.9)$ & $142(63.7)^{\mathrm{a}}$ \\
\hline Infratentorial ICH, $n$ (\%) & $12(15.6)$ & $19(7.9)$ & $8(14.8)$ & $23(8.8)$ & $13(17.3)$ & $18(7.5)$ \\
\hline Ventricular irruption, $n$ (\%) & o) $44(57.1)$ & $119(49.8)$ & $32(59.3)$ & $131(50.0)$ & $41(54.7)$ & $122(50.6)$ \\
\hline Third ventricle & $32(41.6)$ & $65(27.2)^{a}$ & $25(46.3)$ & $72(27.5)^{a}$ & $30(40.0)$ & $67(27.8)^{a}$ \\
\hline Fourth ventricle & $23(29.9)$ & $55(23.0)$ & $19(35.2)$ & $59(22.5)$ & $22(29.3)$ & $56(23.2)$ \\
\hline Lateral ventricles & $40(51.9)$ & $110(46.0)$ & $29(53.7)$ & $121(46.2)$ & $38(50.7)$ & $112(46.5)$ \\
\hline In-hospital mortality, $n$ (\%) & 6) $43(55.8)$ & $101(42.3)$ & $26(48.1)$ & $118(45.0)$ & $37(49.3)$ & $107(44.4)$ \\
\hline
\end{tabular}

DBP - diastolic blood pressure, GCS - Glasgow coma scale, ICH - intracerebral hemorrhage, MAP-mean arterial pressure, SBP - systolic blood pressure. $a_{p}<0.05$, after Bonferroni correction; for comparison of upper vs. lower quartiles

Table II. Multivariate analysis of factors independently associated with in-hospital mortality in patients with supratentorial hemorrhage $(n=285)$ : a Cox proportional hazards modela

\begin{tabular}{|lcc|}
\hline Variables & Hazard ratio (95\% confidence interval) & Value of $p$ \\
\hline GCS $<8$ at admission & $3.27(2.08-5.14)$ & $<0.001$ \\
\hline Hematoma volume $>70 \mathrm{ml}$ & $2.09(1.24-3.54)$ & 0.006 \\
\hline The highest vs. the lowest SBP quartile & $1.19(1.02-1.38)$ & 0.03 \\
\hline Age, per one year increment & $1.02(1.00-1.03)$ & 0.01 \\
\hline
\end{tabular}

GCS - Glasgow coma scale, SBP, systolic blood pressure. aAdjusted for gender, age, extension into the ventricles (any or separate), diastolic and mean blood pressure at hospital admission, ICH location (supratentorial vs. infratentorial; deep vs. lobar; and cerebellar vs. brainstem) and surgical interventions (surgical evacuation and ventriculostomy). Only significant predictors are shown 

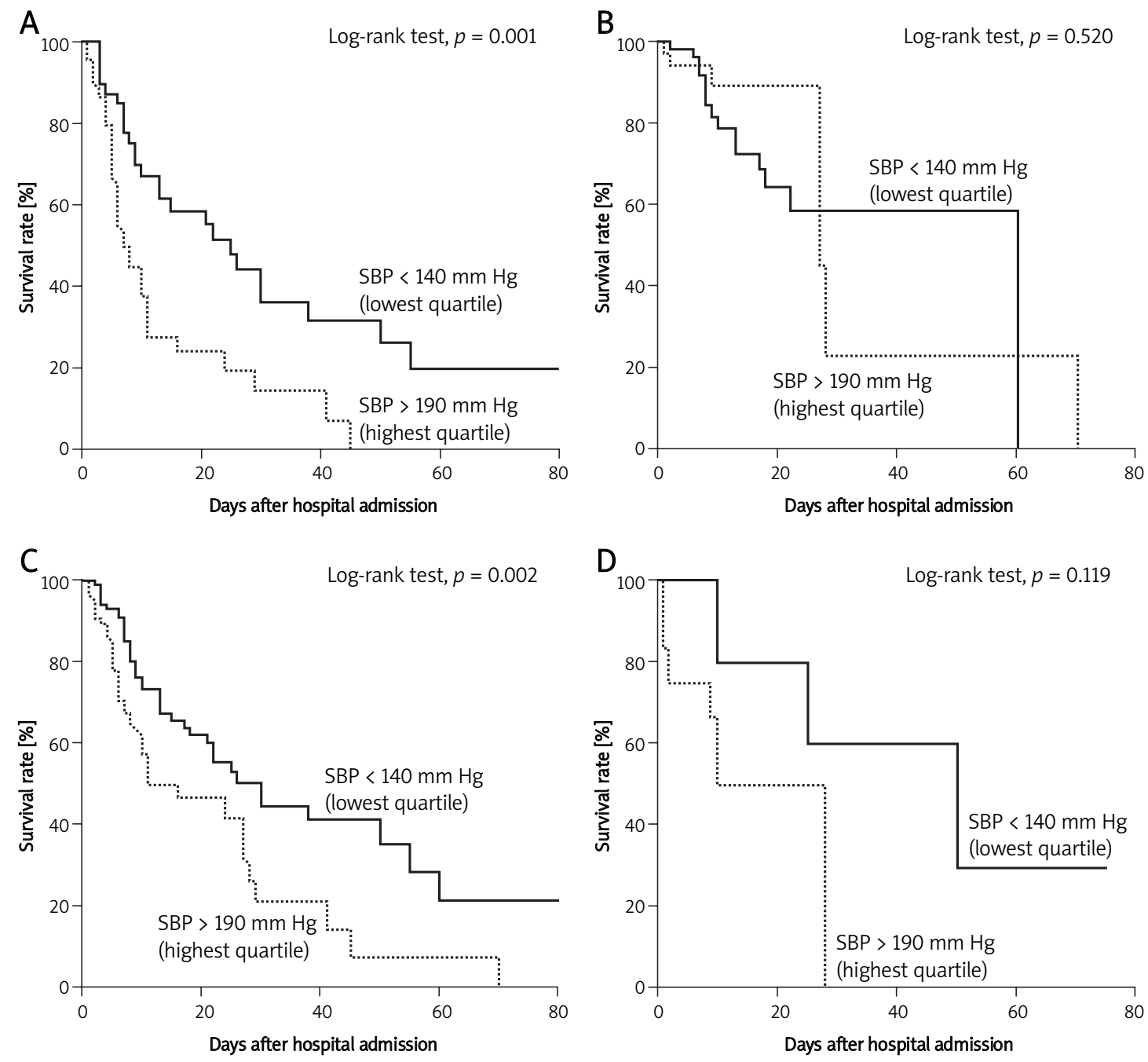

Figure 1. Kaplan-Meier estimates of short-term survival after primary intracerebral hemorrhage (ICH), according to the first systolic blood pressure (SBP) reading at hospital admission, in patients with $(n=163)(\mathrm{A})$ and without $(n=153)$ (B) intraventricular hematoma extension, as well as with supratentorial $(n=285)(C)$ and infratentorial $(n=31)(\mathrm{D}) \mathrm{ICH}$

dictors of short-term outcome (Table II). The association of a high SBP with mortality remained significant only for supratentorial ICH and in cases with hematoma extension into the ventricular system at first brain imaging (Figure 1). We also explored the possibility that low BP levels would be associated with a high mortality, together with very high BP readings (i.e., a "J-pattern relationship" between BP levels and mortality frequency) either as quartiles, quintiles or intuitive BP cutoffs (Figure 2$)$ in the whole cohort $(n=316)$. A J-shaped relationship was observed for SBP intervals and case fatality rate, but not for DBP or MAP (Figure 2).

\section{Discussion}

We found that a high BP at hospital admission is associated with an increased probability of $\mathrm{ICH}$ extension into the ventricular system and with

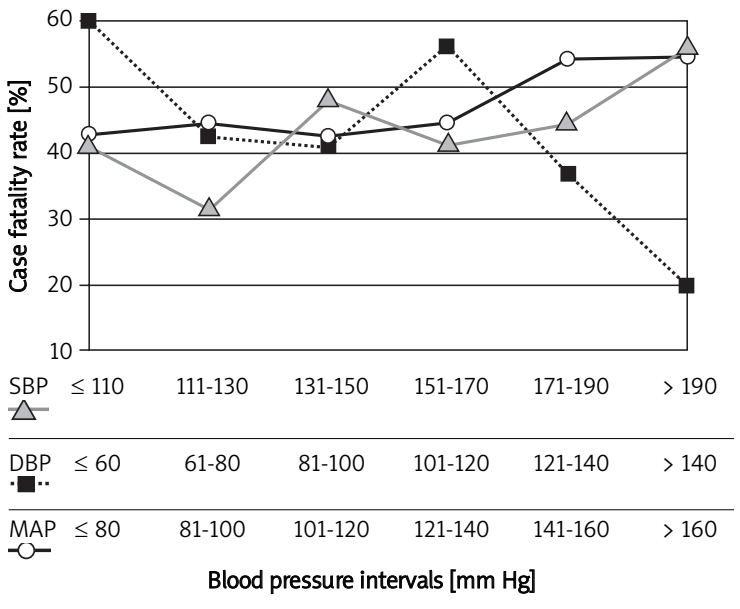

Figure 2. Case fatality rate according to blood pressure intervals (as $20 \mathrm{~mm} \mathrm{Hg}$ increments) $D B P$ - diastolic blood pressure, SBP - systolic blood pressure, $M A P$ - mean arterial pressure 
a high short-term mortality in patients with supratentorial ICH. The effect of a high BP is possibly mediated by a high frequency of extension into the ventricular system, especially the third ventricle. We observed a high acute case fatality rate, possibly due to the fact that all patients analyzed in this study were managed in the general wards.

Several autonomic responses have been described after acute cerebrovascular disease, including ICH $[2,6,13]$. The association of a high BP at hospital admission and early outcome after $\mathrm{ICH}$ has been discussed previously [2, 8-11], but the nature of this relationship is difficult to explain. It has been suggested that acute hypertension after ICH may facilitate hematoma enlargement, perihematoma edema or rebleeding, all dynamic factors associated with outcome [7]. Hematoma expansion is a strong predictor of short-term outcome [14, 15], but this factor has not been consistently associated with a high BP at hospital admission [16-19]. Alternatively, an elevated BP could be an epiphenomenal marker of early deterioration and not the cause of the neurologic impairment [20].

Clinical and experimental studies have shown that SBP increases acutely after ICH or cerebral infarction, with a natural tendency to decrease in the following days [2, 6, 21-24]. Impaired cerebrovascular reactivity [25] and baroreflex dysfunction [26] can result from ICH or cerebral infarction, which could mediate in part the acute BP response after brain injury and intracranial hypertension. Recent trials $[27,28]$ have failed to demonstrate a reduction in mortality with strict $\mathrm{BP}$ control in the acute state after $\mathrm{ICH}$. This suggests that there is a tight balance that needs to be maintained between adequate cerebral perfusion after $\mathrm{ICH}$ [6] and the risk of hematoma expansion imposed by a high systemic blood pressure [16], which highlights the importance of adequate patient selection for strict blood pressure control in the acute management of $\mathrm{ICH}$. Our results confirm the notion that patients with supratentorial ICH experience more benefit from tight BP control, as compared with those with brainstem or cerebellar hematomas.

In the mid and long term, a J-shaped relationship has been described for BP levels (below 110/70 $\mathrm{mm} \mathrm{Hg}$ and above 140/90 $\mathrm{mm} \mathrm{Hg}$ ) with cardiovascular outcomes [1, 29]. This has challenged the "lower the better" concept in BP management. Moreover, in the acute presentation of the different forms of cerebrovascular disease (ischemic stroke, $\mathrm{ICH}$, subarachnoid hemorrhage and cerebral venous thrombosis) the first SBP readings (but not DBP or MAP) at hospital admission also show a J-shaped relationship with short-term mortality, so that the risk of death is highest for SBP $<100 \mathrm{~mm} \mathrm{Hg}$, decreases between $100 \mathrm{~mm} \mathrm{Hg}$ and $139 \mathrm{~mm} \mathrm{Hg}$, and gradually reaches a new zenith at SBP > $220 \mathrm{~mm} \mathrm{Hg}$ [2].
In the present report we confirm this pattern in an independent population; however, here we observed very few $(n=3)$ cases with SBP $<100 \mathrm{~mm} \mathrm{Hg}$, and therefore Kaplan-Meier and Cox analyses did not show a significant association for low SBP levels and short-term outcome. On the other hand, the present study was about the first BP readings at hospital admittance and not about BP control in relation to outcome. A better understanding of BP and organ perfusion dynamics will help to define patient-centered cut-offs for effectively reducing cardiovascular outcomes and mortality [30-32].

This study has several limitations that should be acknowledged. First, no information could be obtained about other relevant prognostic variables, such as blood glucose levels, central temperature, premorbid brain parenchymal characteristics, withdrawal of care and timing of neuroimaging or surgery $[7,14,19]$. Also, no sequential information from brain imaging could be obtained to evaluate hematoma expansion, secondary intraventricular extension, or perihematoma edema, and the dynamic changes of BP throughout the first week after ICH were not registered. More importantly, we could not eliminate the possibility of hematoma enlargement before the first CT or MRI was performed, because neuroimaging was undertaken at different time points for each patient. Nevertheless, these findings may help to identify patients more suitable to experience benefit from strict BP control in the acute phase of $\mathrm{ICH}$. Whether aggressive reduction of $\mathrm{BP}$ will result in better outcomes is yet to be established $[27,28]$.

In conclusion, a high BP is associated with an adverse short-term outcome. High BP readings are more related to irruption into the ventricular system than with hematoma volume, therefore influencing mortality risk. However, a significant proportion of deaths could not be explained by ventricular irruptions, which highlights the need for more studies to define adequate BP cutoffs to positively affect cardiovascular outcomes in patients with hypertension [30-32].

\section{References}

1. Banach M, Bhatia V, Feller MA, et al. Relation of baseline systolic blood pressure and long-term outcomes in ambulatory patients with chronic mild to moderate heart failure. Am J Cardiol 2011; 107: 1208-14.

2. Baños-González M, Cantú-Brito C, Chiquete E, et al. Systolic blood pressure and functional outcome in patients with acute stroke: a Mexican registry of acute cerebrovascular disease (RENAMEVASC). Arch Cardiol Mex 2011; 81: 169-75.

3. Ruiz-Sandoval JL, Romero-Vargas S, Chiquete E, PadillaMartínez JJ, González-Cornejo S. Hypertensive intracerebral hemorrhage in young people: previously unnoticed age-related clinical differences. Stroke 2006; 37: 2946-50. 
4. Chiquete E, Ruiz-Sandoval MC, Alvarez-Palazuelos LE, Padilla-Martínez JJ, González-Cornejo S, Ruiz-Sandoval JL. Hypertensive intracerebral hemorrhage in the very elderly. Cerebrovasc Dis 2007; 24: 196-201.

5. Ruiz-Sandoval JL, Chiquete E, Gárate-Carrillo A, et al.; RENAMEVASC investigators. Spontaneous intracerebral hemorrhage in Mexico: results from a Multicenter Nationwide Hospital-based Registry on Cerebrovascular Disease (RENAMEVASC). Rev Neurol 2011; 53: 705-12.

6. Garg RK, Liebling SM, Maas MB, Nemeth AJ, Russell EJ, Naidech AM. Blood pressure reduction, decreased diffusion on MRI, and outcomes after intracerebral hemorrhage. Stroke 2012; 43: 67-71.

7. Morgenstern LB, Hemphill JC 3rd, Anderson C, et al.; American Heart Association Stroke Council and Council on Cardiovascular Nursing. Guidelines for the management of spontaneous intracerebral hemorrhage: a guideline for healthcare professionals from the American Heart Association/American Stroke Association. Stroke 2010; 41: 2108-29.

8. Dandapani BK, Suzuki S, Kelley RE, Reyes-Iglesias Y, Duncan RC. Relation between blood pressure and outcome in intracerebral hemorrhage. Stroke 1995; 26: 21-4.

9. Carlberg B, Asplund K, Hägg E. The prognostic value of admission blood pressure in patients with acute stroke. Stroke 1993; 24: 1372-5.

10. Fogelholm R, Avikainen S, Murros K. Prognostic value and determinants of first-day mean arterial pressure in spontaneous supratentorial intracerebral hemorrhage. Stroke 1997; 28: 1396-400.

11. Terayama Y, Tanahashi N, Fukuuchi Y, Gotoh F. Prognostic value of admission blood pressure in patients with intracerebral hemorrhage. Keio Cooperative Stroke Study. Stroke 1997; 28: 1185-8.

12. Ruiz-Sandoval JL, Chiquete E, Romero-Vargas S, PadillaMartínez JJ, González-Cornejo S. Grading scale for prediction of outcome in primary intracerebral hemorrhages. Stroke 2007; 38: 1641-4.

13. Amin $\mathrm{H}$, Aronow WS, Lleva $\mathrm{P}$, et al. Prevalence of transthoracic echocardiographic abnormalities in patients with ischemic stroke, intracerebral hemorrhage, and subarachnoid hemorrhage. Arch Med Sci 2010; 6: 40-2.

14. Davis SM, Broderick J, Hennerici M, et al.; Recombinant Activated Factor VII Intracerebral Hemorrhage Trial Investigators. Hematoma growth is a determinant of mortality and poor outcome after intracerebral hemorrhage. Neurology 2006; 66: 1175-81.

15. Demchuk AM, Dowlatshahi D, Rodriguez-Luna D, et al.; PREDICT/Sunnybrook ICH CTA study group. Prediction of haematoma growth and outcome in patients with intracerebral haemorrhage using the $\mathrm{CT}$-angiography spot sign (PREDICT): a prospective observational study. Lancet Neurol 2012; 11: 307-14.

16. Ohwaki K, Yano E, Nagashima H, Hirata M, Nakagomi T, Tamura A. Blood pressure management in acute intracerebral hemorrhage: relationship between elevated blood pressure and hematoma enlargement. Stroke 2004; 35: 1364-7.

17. Jauch EC, Lindsell CJ, Adeoye O, et al. Lack of evidence for an association between hemodynamic variables and hematoma growth in spontaneous intracerebral hemorrhage. Stroke 2006; 37: 2061-5.

18. Fujii Y, Takeuchi S, Sasaki O, Minakawa T, Tanaka R. Multivariate analysis of predictors of hematoma enlargement in spontaneous intracerebral hemorrhage. Stroke 1998; 29: 1160-6.
19. Lou M, Al-Hazzani A, Goddeau RP Jr, Novak V, Selim M. Relationship between white-matter hyperintensities and hematoma volume and growth in patients with intracerebral hemorrhage. Stroke 2010; 41: 34-40.

20. Passero S, Ciacci G, Rossi S. Blood pressure rise in spontaneous intracerebral haemorrhage: epiphenomenon or precipitating factor? J Hum Hypertens 2003; 17: 77-9.

21. Wallace JD, Levy LL. Blood pressure after stroke. JAMA 1981; 246: 2177-80.

22. Jørgensen HS, Nakayama H, Christensen HR, Raaschou HO, Kampmann JP, Olsen TS. Blood pressure in acute stroke. The Copenhagen Stroke Study. Cerebrovasc Dis 2002; 13: 204-9.

23. Dai J, Li S, Li X, Xiong W, Qiu Y. The mechanism of pathological changes of intraventricular hemorrhage in dogs. Neurol India 2009; 57: 567-77.

24. lida S, Baumbach GL, Lavoie JL, Faraci FM, Sigmund CD, Heistad DD. Spontaneous stroke in a genetic model of hypertension in mice. Stroke 2005; 36: 1253-8.

25. Diedler J, Sykora M, Rupp A, et al. Impaired cerebral vasomotor activity in spontaneous intracerebral hemorrhage. Stroke 2009; 40: 815-9.

26. Sykora M, Diedler J, Poli S, Rupp A, Turcani P, Steiner T. Blood pressure course in acute stroke relates to baroreflex dysfunction. Cerebrovasc Dis 2010; 30: 172-9.

27. Anderson CS, Huang $\mathrm{Y}$, Arima $\mathrm{H}$, et al.; INTERACT Investigators. Effects of early intensive blood pressurelowering treatment on the growth of hematoma and perihematomal edema in acute intracerebral hemorrhage: the Intensive Blood Pressure Reduction in Acute Cerebral Haemorrhage Trial (INTERACT). Stroke 2010; 41: 307-12.

28. Qureshi Al, Palesch YY, Martin R, et al.; Antihypertensive Treatment of Acute Cerebral Hemorrhage Study Investigators. Effect of systolic blood pressure reduction on hematoma expansion, perihematomal edema, and 3month outcome among patients with intracerebral hemorrhage: results from the antihypertensive treatment of acute cerebral hemorrhage study. Arch Neurol 2010; 67: 570-6.

29. Banach M, Michalska M, Kjeldsen SE, Małyszko J, Mikhailidis DP, Rysz J. What should be the optimal levels of blood pressure: does the J-curve phenomenon really exist? Expert Opin Pharmacother 2011; 12: 1835-44.

30. Barylski M, Małyszko J, Rysz J, Myśliwiec M, Banach M. Lipids, blood pressure, kidney - what was new in 2011? Arch Med Sci 2011; 7: 1055-66.

31. Banach M, Aronow WS. Hypertension therapy in the older adults-do we know the answers to all the questions? The status after publication of the ACCF/AHA 2011 expert consensus document on hypertension in the elderly. J Hum Hypertens 2012; 26: 641-3.

32. Stępień M, Wlazeł RN, Paradowski M, et al. Serum concentrations of adiponectin, leptin, resistin, ghrelin and insulin and their association with obesity indices in obese normo- and hypertensive patients - pilot study. Arch Med Sci 2012; 8: 431-6. 